\title{
FAMILISMO Y PATRONAZGO COMO SISTEMAS DE PODER LOCAL EN CERDEÑA
}

\author{
Y. Michal Bodemann \\ (University of Toronto, Canadá)
}

Gran parte de la literatura antropológica de América Latina y del Mediterráneo en particular se halla preocupada por la identificación de sistemas insulares de patronazgo y la individualización de los agentes del poder. En el artículo que sigue se muestra como es más provechoso el concebir a esos «patrones» o «agentes del poder» en el marco de una élite local y por lo tanto como representantes de las clases rurales altas. Para el caso de la Cerdeña actual, esta éfite local está definida por cuatro características: se halla atada al área local por medio de lazos particulares de parentesco; se halla atada al Estado por medio de posiciones que ocupan algunos de sus miembros en determinados cuerpos políticos o cuasi-políticos; tiende a monopolizar el acceso a dichos cuerpos, y la élite local asume funciones económicas básicas en las diferentes fases del capitalismo. El siguiente caso de análisis intenta demostrar estas características con algún detalle. En la ptimera sección se intenta reconstruir la élite local del pueblo de Orgosolo alrededor del año 1910, sobre la base de un informe sobre una lucha encarnizada entre familias en el lugar y se relacionan los hallazgos de Orgosolo con datos acerca de una comunidad de vecinos. En la segunda sección del ensayo, esta antigua élite local es comparada con la nueva élite local que surge en la década de 1960 . $^{\prime}$

1. Me gustaría expresar mi agradecimiento a Giovanni Arrighi, Stephen Berkowitz, Jesús M. de Miguel, Susan Berkowitz, Anton Blok, John Day, Claude Meillassoux, Mariano Muggianu, Miguel Murmis, Robin Ostow, Giovanni Andrea Tegas, Barry Weliman, e Irving Zeitlin por sus observaciones a un bosquejo inicial y/o sus útiles sugerencias a esta investigación. 
«Los sociólogos no han prestado una atención profunda a la pequeña ciudad como estructura de poder, mucho menos en un sistema de poder que se extiende por toda la nación.»

C. Wright Mills ${ }^{2}$

\section{La «disamistade» de Orgosolo (1903-1917)}

En 1903 se pusieron en matcha en la Barbagia una serie de acontecimientos dramáticos y terroríficos. ${ }^{3}$ Estos hechos mantuvieton en suspenso a toda Cerdeña y despertaron fuertes emociones en el resto de Italia por más de una década. Me estoy refiriendo a la disamistade de Orgosolo, una lucha familiar encarnizada en un pueblo de pastores en la Barbagia, en el curso de la cual fureron asesinadas diecisiete personas, aunque algunas fuentes sitúan en más de veinte el número de víctimas.

Los elementos principales de la disamistade fueron reunidos por el periodista franco-italiano Franco Cagnetta. ${ }^{4}$ En 1903 muere sin dejar descendientes directos un tico pastor terrateniente llamado Diego Moro. La disamistade aparece inicialmente como una disputa por la herencia: a los

2. C. Wright Mills, The Power Elite (Nueva York: Oxford University Press, 1956), pág. 368 .

3. La zona de Barbagia, dividida a su vez en distintas tegiones, es el área que rodea a las montañas Gennargentu, la cadena montañosa más elevada de la isla (cerca de 1.800 mettos), en la parte central de Cerdeña. La mayot parte de esta zona se halla en la provincia de Nuoro y su economía es fundamentalmente pastorii. Actualmente, estas comunidades densamente agrupadas poseen una población que oscila entre los mil y los seis mil habitantes.

4. VÉase la obra de Franco Cagnetta, Banditi à Orgosolo (Rimini-Firenze: Guafaldi, 1975). Cagnetta teconstruyó el caso sobre la base de informes policiales, noticias aparecidas en los periódicos de fa época y entrevistas efectuadas en Orgosolo. Tanto los informes policiales como así también las entrevistas deberían ser considerados con prudencia. Los informes provenientes de la comunidad, con una causa 
80 años, después de la muerte de su esposa, Diego Moto tecibe en su casa a una sobrina, quien está casada y tiene varios hijos, que cuidará de él en los últimos años de su vida. ${ }^{5}$

Diego Moro dejó en testamento toda su propiedad —una tierra valorada en aptoximadamente 300.000 dólares (de 1975) y una suma similar en efectivo - en cuatro partes iguales a los hijos de su hermana, uno de los cuales era Giovanna Corraine. La herencia equitativa y divisible es un importante principio sardo pero las complicaciones en este caso surgieron porque los beneficiarios no exan sus propios hijos y porque la riqueza de Moto era «corrupta». El dato más importante es que uno de los beneficiarios, su sobrina Giovanna Corraine, estaba cuidando de él, ella y su familia vivían en casa de Diego Moto en el momento de su muerte y por lo tanto ella estaba más estrechamente vinculada a la propiedad que cualquiera de los otros beneficiarios. Había además un desequilibrio político muy claro entre los cuatro hermanos. El hijo de Giovanna, Diego Cossu, era sacerdote y oficiaba en la iglesia del pueblo; como tal, tenía un acceso privilegiado al poder político en Nuoro, una ciudad importante a seis $u$ ocho horas de caballo del pueblo.

La disamistade comenzó con la acusación de un hermano de Giovanna, Giovanni Antonio, en el sentido de que eila estaba ocultando las riquezas

proselitista como en este caso, tienden a prejuiciarse en un sentido u otro; la gente se muestra reacia a hablar francamente sobre dichos temas ya que los considera "cuestiones familiares"; los informes policiales y las noticias aparecidas en los periódicos - dado el abismo cultural entre comunidades como Orgosolo y el mundo exterior- distotsionan los hechos aún más. Para los propósitos que nos ocupan en el presente caso, a pesar de algunas lagunas, el relato de Cagnetta sigue siendo muy satisfactorio. Informes concisos, a mi juicio totalmente fieles, con respecto a la disputa familiar y opuestos a lo sostenido por Cagnetta en el sentido de que el pueblo respaldaba totalmente a los Corraine, aparecieron en el Giornale d'Italio en octubre de 1913. Algunos de estos informes forman parte de una extensa nota en la obra de Vilfredo Pareto, Traité de Sociologie Génerale (París-Lausanne: Librairie Payot, 1919), vol. 2, pp. 1389 y ss.

5. Existe algún interrogante con respecto al origen de la riqueza de Moto. Cagnetta señala que se hizo rico robando a un hombre sumamente adizerado en una ciudad costera y engañando a sus socios en el robo. Sin embargo, Diego Moro era un hombre joven cuando se estaba aboliendo el sistema feudal en Cerdeña -una época en la que muchos individuos -mpleaban prácticas crueles y deshonestas a fin de hacerse con las tierras comunales- y él o su familia bien pudieron haber tomado parte en estos robos. Por otra parte, se casa con Ia hija del hermano de su padre, Luigia Moro, a quien se señalaba como una persona adinetada. Yo creo, por lo tanto, que es proba. ble que su riqueza es anterior a estos actos de pillaje y que él pertenecia a una familia de dinero, una suposición corroborada por el hecho de que desposa a una prima carnal, una práctica común en la Barbagia y destinada a mantener la propiedad como bien común. 
de Diego Moro y que había tomado posesión de facto de la hetencia de éste. La aliada de Giovanni Antonio en esta disputa era su hermana An. gela Rosa; juntos constituían el núcleo del «bando Corraine». La aliada de Giovanna era la otra hermana, Domenica; estas dos hermanas constituían el «bando Cossu». Para poner fin a la disputa, Giovanni Antonio Corraine propuso una solución que era una práctica aceptada en el su connotiu, una ley sarda habitual. Él propuso un doble matrimonio de uno de sus hijos y una de sus hijas con una de las hijas y uno de los hijos de Giovanna respectivamente. Don Diego, el sacerdote, como portavoz del «bando Cossu» rechazó esta proposición debido a la estrecha relación de parentesco, aún cuando en Cerđeña los casamientos entre primos catnales no son de ningún modo extraños.

Con el rechazo de esta proposición comenzó la lucha familiar propiamente dicha: al principio fue la matanza de algunas reses pertenecientes a los Cossu, presumiblemente a cargo de los Corraine, luego, a modo de venganza se produjo el asesinato de uno de los hijos de Giovanni An. tonio, Carmine, a manos de uno de los hijos de Domenica, Egidio Podda. Desde ese momento sobrevino una cadena de sangrientas venganzas entre los dos bandos en conflicto. En el curso de la disamistade surgieron dos cuestiones importantes: el «bando Cossu», con Don Diego, tenía a los representantes del Estado plenamente de su parte, especialmente los carabinieri y los tribunales, hasta el extremo de que Egidio Podda, aunque claramente responsable de la muerte de Carmine, fue absuelto de todos los cargos. En 1913 los Cossu incluso se las arreglaron para hacer artestar a 52 Corraine, prácticamente la totalidad de los que no habian buido a las montañas. Por otra parte, ninguno de los Cossu había caído en prisión. Los Corraine, entonces, a pesar de que inicialmente habian confiado en los organos oficiales del Estado a nivel de procedimientos «ordenados», se dieron cuenta pronto de que el Estado estaba absolutamente de parte del bando Cossu.

La segunda cuestión importante que afloró del conflicto fue que la disamistade incluía continuamente nuevos aliados en ambas partes -aliados que eran parientes de las partes en conflicto, es decir, vinculados por matrimonio o que eran hijos de los hermanastros. Por lo tanto, la disamistade no se circunscribió a un pequeño grupo de patientes y amenazó con involucrar a estratos cada vez más amplios de la comunidad. En el curso de su guerra combinada contra los Cossu y las fuerzas policíacas atiadas, los Corraine dieron origen a una banda de delincuentes populates, la banda Succu, quienes pronto fueron conocidos como la banda más despiadada que jamás había existido en la isla. Cagnetta escribe: 
«Teniendo en cuenta las cifras, se podría decir que en aquella lucha entre malvivientes y policías, los primeros salieron victoriosos; el Estado, a pesat del número de sus soldados y de la eficacia de los medios a su alcance no había logrado, ante la evidencia de los hechos, ni proteger la integridad física de los miembros del bando Cossu ni eliminar en persona a sus mayores enemigos, los evadidos del bando Corraine. $\rangle^{6}$

La situación llegó a un punto en que los Cossu, dada la impotencia del Estado frente a una amenaza creciente de los Corraine y de la banda Succu, se vieron obligados a iniciar negociaciones que culminaron exitosamente en el verano de 1916. Al banquete celebrando la paz asistieron los líderes de los dos bandos antagónicos, don Diego Cossu y el bandido Ono. ratu Succu, como así también el prefecto de la provincia, el obispo, el miembro del Parlamento en Roma y un grupo de personalidades de la tegión. Algunos días más tarde el contingente de la policía especial se retiró de Orgosolo y, en 1917, en el juicio contra diez miembros de la banda Succu y de los Corraine, a los cuales se acusaba de haber cometido entre tres y quince asesinatos y numerosos cargos adicionales, los diez acusados fueron absueltos «per non aver commesso $i$ fatti» (por no haber cometido tales hechos), siendo esencialmente una admisión por parte del Estado de una derrota total frente a la arcaica sociedad local.

Este detallado repaso de la disamistade de Orgosolo es útil porque este caso revela más efectivamente de lo que cualquier estudio antropológico podría hacerlo, algunos de los rasgos importantes de la estructura social Barbaricina a comienzos de siglo.

Familismo amoral y exclusivista. En primer término, surgen algunos puntos importantes en relación al parentesco y la estructura familiar. En la superficie parece sustentar la idea de Banfeld con respecto al familismo amoral, de acuerdo con la cual Montegranesi (y presumiblemente otros mediterráneos «subdesarrollados» también) «maximiza la ventaja material y a corto plazo de la familia nuclear»: Giovanna Corraire, inicialmente, reclama las riquezas de su tío para su familia y para la familia de su hija. Sin embatgo, en el curso de la sangrienta disputa familiar, se vuelve evidente precisamente el principio opuesto. Ambos bandos teciben el apoyo de las familias de otros hermanos y, a medida que el conflicto se extiende, cada vez más primos, parientes políticos $y$ otros parientes brindan su

6. Op. cit., p. 213 . 
apoyo al bando con el cual se hallan vinculados por parentesco y lo hacen de tal modo que ellos mismos se convierten en blanco de los asesinatos, evidentemente un precio muy alto el que pagan por cualquier «ventaja material» que puedan obtener como recompensa. Un estudio del Gráfico I revela que los grupos de parentesco más periférico no se encuentran de ninguna manera menos involucrados en la lucha que los grupos más estre. chamente ligados. A juzgar por el númeto de muertes y la activa participación de tales grupos, como la banda Succu, estos grupos «periféricos» no se hallan menos implicados en la sangrienta disputa que aquelios que iniciaron la disamistade. Esta observación no sólo contradice la teoría de Banfield acerca del familismo, sino que también contrasta agudamente con las ideas del sociólogo sardo Luca Pinna, quien substituye «exclusivo» por «amoral» y llega a la conclusión de que la estructura sarda de parentesco se halla gobernada por un «familismo exclusivista» al que define como: "Aquella tendencia, evidenciada en la búsqueda, que lleva a nuestro hipotético núcleo familiar a manifestar un rechazo radical de las "buenas relaciones" que superan el propio ámbito y, por lo tanto, las "buenas relaciones" comunitarias." ${ }^{7}$

En este sentido es importante observar más detenidamente algunas de las características de la estructura de parentesco tal como surgen del relato de la disamistade. La disputa se origina a partir de un conficto de los cuatro hermanos casados. En consecuencia aparece que -y ello está plenamente sustentado por mis propios datos reunidos en el vecindario Telemula ${ }^{8}$ - la solidaridad de los hermanos es precaria y substituida por la solidaridad de los varones fuera de sus grupos de parentesco. Por ejemplo, es notable que sea el hermano varón, Giovanni Antonio Corraine, quien inicie la disputa familiar con su acusación de fraude en la herencia; es el hijo politico de Giovanna, Antonio Piredda, quien se transforma en un activo partidario del bando Cossu; Michele Manca y su familia ${ }^{9}$ se alía con sus futuros parientes, los Devaddis; los Succu del bando Cossu junto a los Pisanu, etc., y sobre todo el primer intento de reconciliación, la proposición de matrimonio cruzado, hubiese transformado a Giovanna y

7. Luca Pinna, La famiglia esclusiva: Parentela e clientelismo in Sardegna (Bati: Laterza, 1971), p. 72. Mientras Pinna intenta mantenerse a distancia de la fllosofía de la cultura de la pobreza de Banfield, es en extremo evidente que no rompe con esta propension. El escribe: «El exclusivismo familiar consiste, por lo tanto, tal como nosotros lo entendemos, en la tendencia de las personas a valorat al máximo las cosas propias, desvalorizando al máximo las de los demás." Ibidem, p. 75.

8. El nombre es ficticio.

9. Onotato Succu, líder de la banda Succu, se casó posteriormente con Serafita Manca, hermana de Michele Manca. 


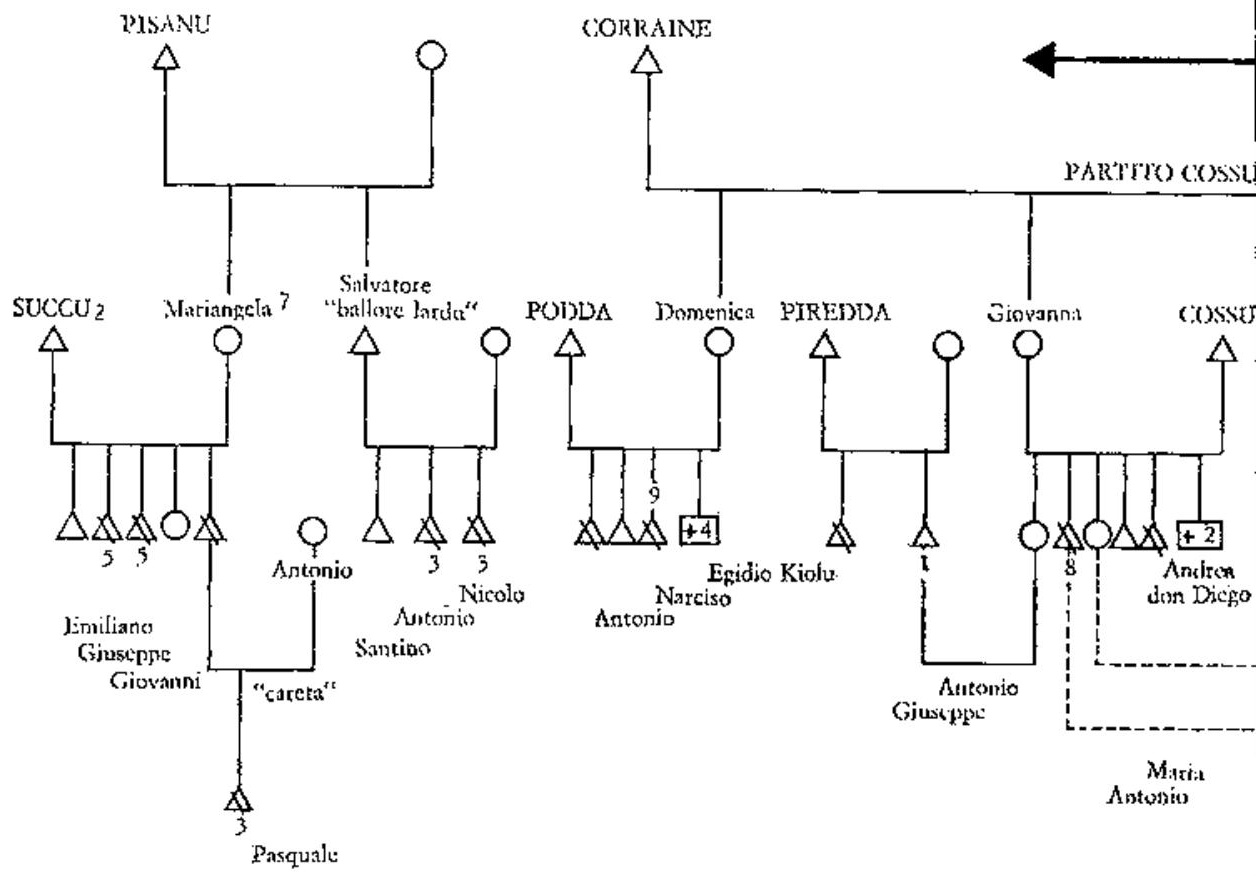

Notis:

Díctimins de lit lercha familiar.

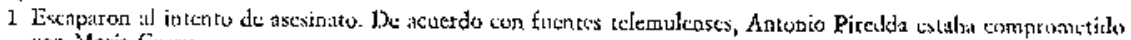
cols Maria Cossu.

2 "Stajpmo in Anserica" (fuente telemolense)

3 Mvlolescentes:

4 P:irina del ()arratklku Midycle Pilounc

5 Muertes en un triple asesimato junto a Pikone

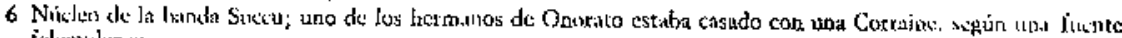

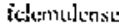

7 "Interessato per ragioni di socictá di bestimae a non conseguire le paci" (Cagnctta, príg. 208)

8 litzos afincs il bando Corraine (detaldes desconocides)

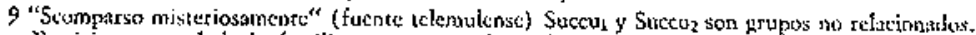

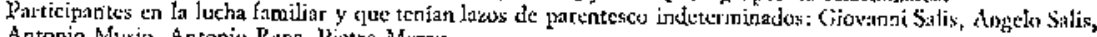
Antorio Musito, Antonio Rana, pistro Mereu 
Giovanni Antonio, hermanos enemigos, en hermanos vinculados entre sí por los lazos más fuertes de parentesco. Es esta fuerte solidaridad inherente a la telación de parentesco de la esposa -ucuyas bases económicas no pueden ser analizadas en este punto- la que la asegura típicamente, la estructura familiar sarda no es exclusiva, sino abierta.

El parentesco como superestructura dominante. El segundo punto que surge de este relato de la disamistade es que dentro de la propia comunidad la superestructura político-legal está regida por lazos de parentesco. Sin embargo, debido a lo fragmentario de los datos esta situación no puede ser documentada en profundidad; es evidente que, internamente, las solidaridades que emergen se hallan basadas fundamentalmente en lazos de parentesco. Por ejemplo, es digno de mención el hecho de que los Oarrakkos — habitualmente jóvenes pastores reclutados de otros pueblos y siempre virtualmente sin relación alguna con los propietarios del rebaño-. no se vieron involuctados en la sangrienta reyerta familiar. ${ }^{10}$

En el marco de este ensayo no será posible entrar en detalle en el problema de por qué son tan dominantes los lazos de parentesco en comunidades como la de Orgosolo. He sostenido en alguna otra parte ${ }^{11}$ que, mientras las sociedades dedicadas a la caza y al cuidado de ganado y especialmente las primeras sociedades agrícolas están dominadas por lazos de parentesco, ${ }^{12}$ lo que hallamos en las comunidades Barbaricinas es una forma de involución social, una intensificación regresiva de los lazos de parentesco: en el curso de la destrucción de la economía tradicional y la estructura social en el siglo XIX, se desintegraron otras importantes relaciones y, en consecuencia, los lazos de parentesco volvieron a surgit como el principal principio organizador a nivel local.13

10. La única excepción en este punto es de Michele Pilcone, Oarraku de Francesco Devaddis, quien fue testigo del asesinato de su patrón, fue pagado por los Cossu y posteriotmente asesinado por traidor, junto a dos Cossu asociados con los Soccu. Este caso excepcional, no obstante, sólo sustenta este argumento: Pilcone cambia de bando -algo inconcebible en una lucha parental- y lo hace sobornado.

11. Véase la obra de Y. M. Bodemann, «Natural Development (Natürwwchsig. keit) in Early Society. A Notc to Friedrich Engels Anthropology". (University of Toronto, mimcografiado, 1978.)

12. Claude Meillassoux, «The social organisation of the peasantry: The economic basis of kinship», Journal of Peasant Studies, vol. 1 (1973): 81-90.

13. Por ejemplo, aquellos de los grupos de ancianos que habían satisfecho funciones cetemoniales y políticas importantes, o aquellos de solidaridades laboralmente orientadas tales como el barracellato, una fuerza policial de los pastores basada en la comunidad. Sobre el barracellato, véase la obra de Russell King, «Some spatial and 
La élite local y las instituciones del Estado. El tercer punto que se desprende claramente de este caso es la naturaleza de la élite local y su relación con las instituciones del Estado. En primer lugar, es evidente que en el momento de la disamistade es la familia de Giovanna y su dependencia parenteral la que constituye la élite local y por lo tanto aparece como el vínculo entre el pueblo y el Estado. Sus hijos y sus parientes, como los Piteddas, mantienen una buena telación con los carabinieri locales, los tribunales y la prefectura. Aparte de una posible transmisión de este status a través de su tío Diego Moro, este papel como élite local adquiere consistencia merced al vínculo con la Iglesia en la persona de su hijo Diego Cossu, el sacerdote del pueblo.

Cuando en este punto describo el papel de los Cossu como élite local quiero significar que se encuentran en una posición en la que monopolizan el acceso de la comunidad a las instituciones del Estado (y la Iglesia) e, inversamente, que ellos son sus tepresentantes orgánicos ${ }^{14}$ porque, en virtud de su acceso privilegiado a los medios locales y de sus lazos de parentesco, se hallan asegurados en la comunidad y en posición de movilizar al menos algunos segmentos de la comunidad con propósitos políticos. Este monopolio de los lazos estatales está claramente demostrado en los frustrados intentos de Giovanni Antonio Corradine de tratar con instituciones estatales como los carabinieri. Tanto los carabinieri locales como los tribunales se negaron a tratar con él y su bando, es decir, como demandante, y actuaron como fieles aliados del bando Cossu.

Por lo tanto, sólo en este sentido de monopolización de los lazos del Estado podríamos hablar de familismo «exclusivo» o "amoral» -como una característica no del sistema de parentesco en general, sino meramente como de la élite local y es, tal vez, por esta razón también que Giovanni Antonio no podía recibir la parte que justamente le correspondía de la herencia: él y los grupos asociados por parentesco con su familia hubiesen desafiado la hegemonía de Giovanna en Orgosolo.

A partir del análisis del caso de Orgosolo surgen tres principios que a mi juicio son fundamentales para la estructura social de los puebios de Barbaricine a principios de sigio. Estos principios son, tesumidos brevemente:

1. Fuertes vínculos uxoriales --solidaridad establecida entre los va-

environmental aspects of conflict tesolution: Livestock in Sardinia». Tijdscbrift Voor Economiscbe en Sociale Geografie, vol. 65, núm. 6 (1974): 407-413.

14. Véase para este punto la noción de Gramsci acerca de los intelectuales orgánicos y la élite del sur. Trataré esta cuestión en profundidad en un próximo trabajo. 
rones y las familias de sus esposas-- debilitan los vínculos inherentes a la familia de origen de los varones. ${ }^{15}$

2. Antes de la transformación de la economía a mediados del siglo $\mathrm{xxx}$, los modos de producción predominantes (pastoralismo y producción doméstica) determinan básicamente el sistema de parentesco; más tarde, el parentesco comienza a asumir una fuerza propia mediante la que dicta de forma preponderante la política y la economía locales.

3. Surge una élite local que se establece como el vínculo crucial entre la comunidad local y el Estado. Esta élite se caracteriza por tres aspectos: está basada en el parentesco y se halla vinculada por lazos de parentesco a la comunidad en general; ;6 algunos de sus miembros tienden a sentirse personalmente atados a las instituciones estatales y como tales ellos personifican al mismo (como sacerdotes, abogados, diputados, etc.); y la élite monopoliza el acceso a todas las instituciones estatales importantes. Cuando fracasa en este cometido, es probable que se produzca el conflicto entre los grupos antagónicos.

\section{La antigua élite local en Telemula (1875-1945)}

Estos rasgos de la élite local Barbaricina pueden demostrarse claramente en el caso de Telemula, un pueblo vecino a Orgosolo situado en la zegión oriental de las montañas Gennargentu. ${ }^{17} \mathrm{El}$ surgimiento de la élite local de Telemula, el grupo de parentesco de los Sanna, ${ }^{18}$ se hizo evidente en el último cuarto del siglo xix. Al comenzar dicho período, muchas

15. Dicho de otro modo, podríamos afirmar que existen lazos poderosos y positivos entre la madre y las hijas casadas, mientras que la relación padre-hijo está dominađa por la tensión. Esta sítuación se describe en la obra de Bodemann (1978, cap. 4).

16. Los terratenientes no locales, por ejemplo, que se apropian de tierras en la comunidad, a menos que se emparenten con el pueblo no cumplen típicamente una parte activa en la política local y no pueden ser considerados como élite local. Naturalmente, pueden ser políticamente activos a un nivel tegional o provincial.

17. Las dos comunidades son muy diferentes en cuanto a densidad de poblacion: en 1901 Orgosolo tenía una población de 2.725 habitantes, y Telemula una de 610 . Sin embargo, la diferencia en la cantidad de tierra comunal corresponde aproximadamente a la diferencia en población.

18. Todos los nombres propios de los telemulenses citados aquí son ficticios. El término un poco vago de «grupo de parentesco» denotaría aquí a un grupo de familias cooperativas estrechamente ligadas por lazos de parentesco. La noción local de ratza, por contraste, denota a un grupo de parentesco habitualmente trazado patrilinealmente a lo largo de seis o siete generaciones. 
pequefras familias con algunos recursos estratégicos desarrollan un activo modelo de cooperación. La familia Lai-Mureddu, que aparece en el Gráfico 2, posee un pequeño almacén y recibe una doble porción de tierras en la división de las tierras comunales. ${ }^{19}$ La familia Corrias-Balisai-Mongili ${ }^{20}$ se destaca por dos importantes características. La familia estaba estrecha* mente vinculada a la Iglesia y, por esa razón, más tarde recibieron el derecho a las tierras eclesiásticas. Los antepasados de los Sanna, como los de los Lai, ocasionalmente habian asumido posiciones importantes en la comunidad $y$, para las pasturas de invierno, utilizaban tradicionalmente una zona estratégicamente situada cerca de la carretera principal que comunicaba el norte con el sur. Antes de eso la familia Sanna había participado activamente en los asuntos políticos. La familia Bassu-Muscau se destacó por esa misma época porque Michele Bassu, que había abandonado sus estudios en el seminario, fue durante mucho tiempo el único residente en Telemula que sabía leer y escribir, siendo también un consumado especialista en medicina popular. ${ }^{21}$

Estas farnilias, por lo tanto, desarrollan activas relaciones de cooperación mutua que posteriormente se ve cimentada por casamientos interfamiliares que se continuatán a lo largo de varias generaciones. Estos matrimonios interfamiliares, aparte de la obvia exclusión de los extraños en el acceso al capital familiar, contrarrestaba la habitual falta de solidaridad del parentesco patrilineal metced a los positivos lazos uxoriales. Con sus vínculos personales con la Iglesia y su extensa estructura política, los Sanna surgen «naturalmente» como la élite local, en su mayor parte como alcaldes y concejales en el puebio.

La formación del grupo de parentesco de los Sanna, en los años que

19. Hasta el año 1865 todas las tierras, con excepción de las prebendas eclesiásticas, eran de propiedad comunal. Algunas partes de estas tierras comunales, Hamadas vidazzone, exan divididas y distribuidas periódicamente para el sembrado de cereales. Comenzada en 1865 y acabaca en 1881 , gran parte de la tierra previamente destinada a la prođucción de cereales fue subdividida en partes iguales por cada familia y se volvió propiedad privada. Por razones que no están enteramente claras, algunas familias corno ia de los Lai recibió una doble porcion de tierra, aproximadamente seis hectáreas. El padre de Michele Lai (del grupo Lai-Mureddu) había sido anteriormente uno de los trece kapos decina (cabeza de un grupo de diez familias), comparable tal vez al papel que cumpliría un antepasado. El pequeño almacén de comestibles de los Lai-Mureddu se convirtió más tarde en un tabacchi privilegiado, y es transmitido matrilineaimente, siendo hoy operado por el grupo «RPR» (tres hermanos).

20. Anna Corrias se casó primeramente con un Corrias y, después de la muerte de su ptimer esposo, con un Mongili. Un tio matetno de Anna Corrias, y el hijo de ella, eran sacerdotes.

21. Véase la nota 19. 


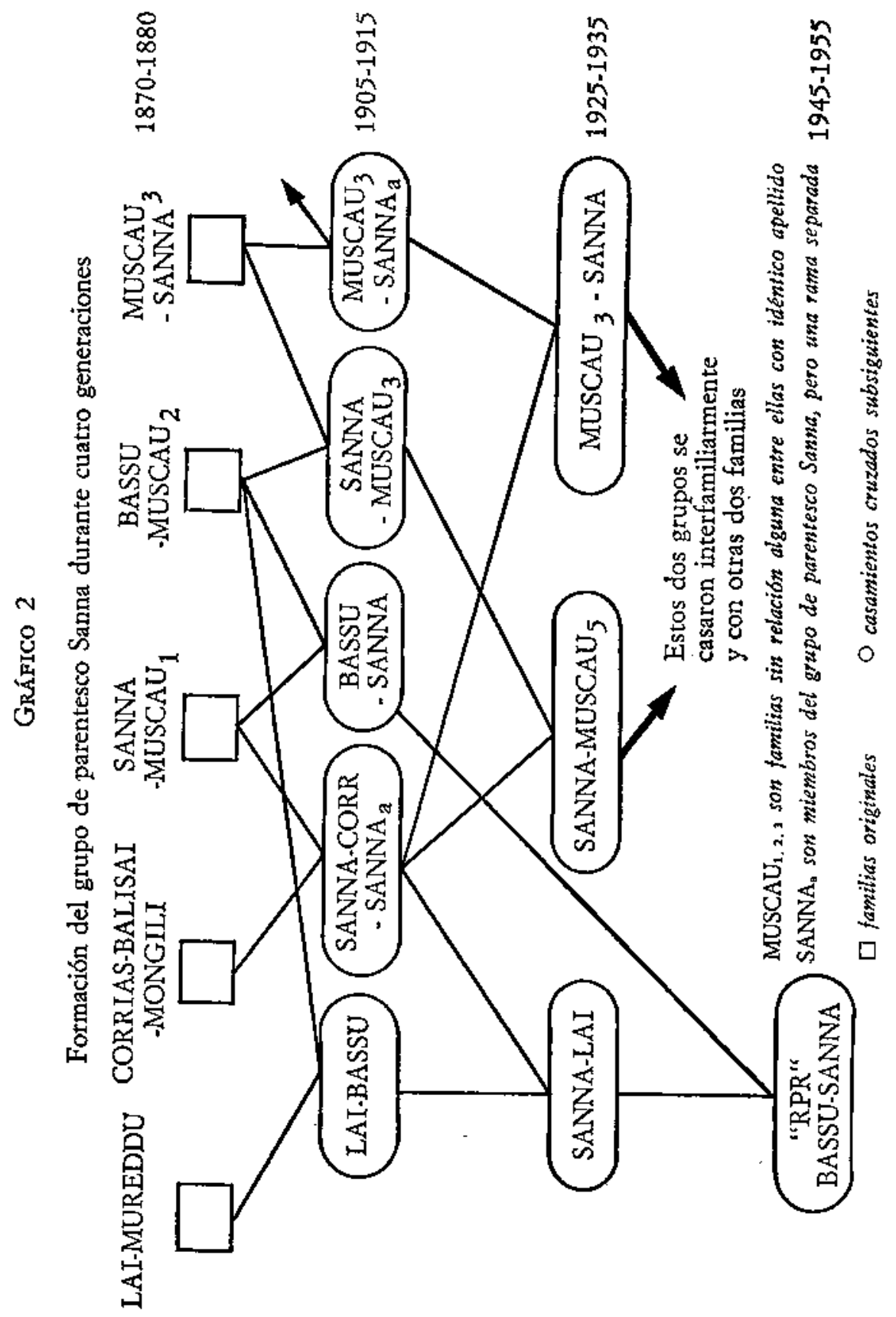


siguieron a la fundación del Estado italiano, se produce en una época en que el mezzogiomo italiano experimenta una seria crisis de su economía y es su población rural, particularmente, la que sufre duras privaciones. En la Barbagia, el drástico aumento de los impuestos conduce a una venta masiva de los recursos locales: a fin de pagar los impuestos las comunidades venden sus bosques de robles, cientos de hectáreas a la vez, a los comerciantes de carbón de leña. Las tierras recientemente parceladas, como las de Telemula, son a menudo expropiadas apenas unos pocos años más tarde porque los nuevos propietarios no podian hacer frente al pago de los impuestos. Está situación, a su vez, alentó a los comerciantes extranjetos a acaparar tierras escogidas para transformarlas en empresas dedicadas a la hacienda.

La división de las zonas de pastoreo invernal y el cambio a usos agricolas de estas zonas, afectó seriamente a la economía pastoril. Los pastores, especialmente, comienzan ahota a saturar con pasturas algunas tierras comunales destinadas a ese fin y son obligados a pagar elevadas rentas en tierras privadas fuera de los límites de la comunidad. Al mismo tiempo, con el desarrollo del sistema moderno de impuestos, se ven obligados a aumentar la producción de queso que, producido anteriormente para el consumo privado y el trueque directo, se transforma ahora en un bien en efectivo.

En la época de esta drástica transformación, los Sanna obtienen un grado de poder político sin precedentes en el pueblo y, esencialmente, mantienen una estricta vigilancia sobre esta transformación económica. Monopolizan el acceso a las autoridades eclesiásticas y cuando, a fines de siglo, se establece un puesto de los Carabinieri Reales, son los Sanna los que alquilan los cuarteles a los carabinieri, lo que a su vez establece una estrecha relación personal entre la fuerza policíaca y los Sanna. Al margen de ser arrendadores de los carabinieri, del Ayuntamiento y de la escuela elemental, la familia acumula cientos de hectáteas de tierra, vendidas a los Sanna por los comerciantes de carbón de leña a maneta de comisiones o recibidas por la familia por otros medios no menos dudosos. Con la ayuda del arciprete Mongili, a un hijo de la familia se le imparte educación médica y una de las hijas llega a ser la primera maestra de la escuela elemental local. ${ }^{22}$

Durante el periodo fascista la economía de Telemula se estanca; las

22. Sin embargo, en el período inmediatamente antexior a la Primera Guerta Mundial se produjo en Telemula una seria tevuelta llamada scioppero. El alcalde en ese momento, un miembro del gxupo de los Sanna y particularmente incompetente y corrupto, es echado físicamente de la ciudad. En las siguientes elecciones se elige como 
principales transformaciones se han producido antes de 1922 y el fascismo se recuerda como una época de severa represión política y de penurias económicas. En consecuencia, desapatece el papel de los Sanna como dirigentes de la transformación económica de la zona, a pesar de que ellos se establecen como los representantes locales del Estado fascista. Tal como muestra el Gráfico 2, los Sanna continuaron con sus casamientos interfamiliares -en términos de lazos de parentesco-m y se aislaron progresivamente del resto de la comunidad. Al mismo tiempo, fracasaton en su intento de vincularse con las élites locales de las comunidades vecinas o con las clases altas de las ciudades principales; se volvieron los "aristó. cratas solitatios» de Telemula. Después de 1945, lentamente comenzaron a perder la iniciativa sobre los asuntos políticos en Telenoula y posteriormente fueron suplantados por una nueva élite local que describiré más adelante.

Un repaso del caso de Telemula con referencia a los datos obtenidos en Orgosolo nos lleva a las siguientes conclusiones:

Primera, sobre una base de comunidad amplia no hay indicio de egósmo familiar como sostiene Banfield, o de exclusivismo, como sustenta Pinna: la gente en general establece nuevos lazos cooperativos y, en virtud de los fuertes lazos de parentesco, pueden surgir tensiones y conflictos de lealtad entre la familia de origen del varón y sus patientes, pero ellos ciertamente hacen imposible la aparición de núcleos familiares aisiados y egoístas. Sin embargo, lo que Banfeld y Pinna realizan es una descripción indudablemente fiel del comportamiento de la élite local en esa época: su amoralidad, el monopolio del poder político y la concentración de las tiquezas se obtiene mayormente por medio de la endogamia, es decir, una estrategia por medio de la cual los lazos de parentesco son «vueitos hacia adentro" y en consecuencia encapsulan a este grupo particular y lo dotan de una fuerte solidaridad interna, si bien lo separan lentamente del resto de la comunidad.

Segunda, con el establecimiento de la élite local, creada por el nuevo

alcalde a uno de los líderes de ia revuelta, desafiando gravemente la posición de los Sanna. Es más, los sentimientos «populistas» de este alcalde lo transforman en un temprano partidario de Mussolini $y$, por lo tanto, superando estratégicamente a los Sanna en los primetos años de fascismo en Italia. Lentamente, no obstante, con el apoyo político masivo desde el exterior y en connivencia con los carabinieri, los Sanna le ganan la mano. El alcalde es expulsado de su despacho, es incriminado con pruebas falsas, huye a las montañas como un delincuente, cae en una trampa tendida por los Sanna y es muerto a tiros por los carabinieri. 
Estado capitalista y basada localmente en el parentesco, el papel del parentesco propiamente dicho cambia de forma significativa. Ya no es más un tipo específico de economía, un modo y una relación específicos de producción que estructuran el sistema de parentesco. Antes bien, se trata de que el parentesco asume ahora un papel principal en la estructuración de la economía; como su superestructura política, asume una fuerza hipostática.

Tercera, en forma muy parecida a la de Orgosolo, en Telemula la élite local también moviliza sus recursos sobre la base del parentesco, tanto dentro del grupo como, a través de sus lazos de parentesco, a la comunidad en general. Además, algunos de sus miembros son representantes personales de las instituciones del Estado y constituyen la personificación de los intereses políticos y económicos del Estado a nivel local..$^{23}$ Finalmente, es importante señalar que en este temprano período la élite local ha hecho abrumador el control de aquelios medios de producción cuya transformación ayuda a producir.

\section{La nueva élite local en Telemula (desde 1965)}

Hasta aquí he estudiado el carácter de la élite rural sarda dentro del contexto histórico específico que sigue a la formación de un Estado capitalista moderno en Italia. Ahora volveré al periodo posterior a 1945 y a un estadio del capitalismo con una caracterización diferente.

En Cerdeña, como en otras zonas del mezzogiorno, este estadio del capitalismo se manifiesta a través del cierre de las minas de carbón locales (a comienzos de la década de 1950), creciente zozobra rural y el establecimiento de la Cassa per il Mezzogiorno, un programa de «modernización» masiva para el sur, con la construcción de nuevas carreteras, el establecimiento de industrias de capital intensivo tales como refinetías de petróleo, una gigantesca fábrica de papel, facilidades para la ampliación del puerto, programas de reforestación y, por último, particularmente visible en el interior rural de la región, una emigtación a gran escala de mano de obra y una afluencia masiva de bienes industriales tales como tejidos, automóviles, aparatos eléctricos, material para la construcción y ciertos alimen* tos, particularmente cerveza y bebidas sin alcohol.

23. Este punto recuerda la formulación de Marx en su Crítica a la Filosofía del Estado de Hegel con referencia al Príncipe. En lugar de ser el Estado la realidad social más alta del individuo, un solo individuo es reproducido como la mayor realidad del Estado. Véase Marx-Engels Werke (Berlín, Dietz: 1843/196), vol. 1, p. 240. 
En este período una nueva élite local, los Taula, comenzó a surgir en Telemula, en forma totalmente independiente de los Sanna. Hacia 1970, este nuevo grupo había logrado en el pueblo un poderío político sólido e indiscutible. He descrito detalladamente el surgimiento de esta nueva élite local ${ }^{24} \mathrm{y}$, por lo tanto, sólo bosquejaré aquí sus rasgos más importantes. Los hermanos Taula, Bruno, Giovanni Antonio, e Iolanda pertenecen a una familia telemulesa típica. Siendo muy pequeños pierden a su padre y son criados por su madre, ayudada por algunos parientes maternos y paternos. La ausencia del padre, quien ha sido pastor como la mayoría de los varones de Telemula, por un lado aleja a los dos varones del medio ambiente pastoril y, por otro lado, al no existir la relación antagónica padre-hijo, los dos mantienen una fuerte solidaridad cooperativa en la casa materna. Los inusuales matrimonios de los dos hijos, además, no fomentaron una fuerte solidaridad parental, ${ }^{25}$ a diferencia de lo ocurrido con Iolanda, quien introdujo a su marido en la esfera de la familia Taula. Debido a una desacos. tumbrada combinación de circunstancias, los hermanos, a los veinte años, se las arreglan para adquirir un camión y son capaces de desarrollar un pequeño negocio de transporte, ya que virtualmente no tienen competencia en Telemula o en los pueblos vecinos. En la década de 1960 construyen un pequeño hotel en Telemula, agregan una moderna panadería, reciben el monopolio del transporte de bebidas embotelladas en una gran zona y, a fines de los años de 1960, abren un negocio de mueblería.

Todas estas actividades no tienen precedentes en Telemula y se los observa con gran desconfianza y, en parte, con abierta oposición. A mediados de la década del sesenta, los Taula evidentemente comprendieron, de forma intuitiva, que sus aventuras comerciales sólo podrían fortalecerse si se las cimentaba sobre alguna forma de poder político en la propia Telemula y comenzaron a establecer una base política en el pueblo.

Entre 1945 y 1970 , la situación política en Telemula eta absolutamente inestable, con cambios permanentes en la administración municipal y con muy pocas personas políticamente preparadas ocupadas en los asuntos locales. ${ }^{26}$ Esto se debió al hecho de que, con la lenta desaparición de

24. Y. M. Bodemann, Telemula: Aspects of the Micro-Organisation of Backwardness in Central Sardinia (University of Toronto: no publicado, 1978); caps. 4 y 5.

25. Bruno se casa con una mujer que no es de Telemula - un hecho algo infrecuente en las comunidades tradicionales- y Giovann Antonio desposa a una mujer perteneciente a una familia pobre, quien fue adoptada de facto por la hermana de la madre de Giovanni. Ni Bruno ni Giovanni desartollaron lazos de dependencia fuertes con las familias de sus esposas.

26. En varias oportunidades un commissario prefettizio, oficial de la prefectura, asumió el control debido a la incompetencia administrativa general, hecho que no debe 
los Sanna, un segmento difuso y heterogéneo de la comunidad, vinculado de diversas formas con la democrazia cristiana, y más tarde también con los socialistas, detentaba el poder nominal de la región. Sin embargo, du. rante gran parte de este período los Sanna - aun cuando habian perdido su iniciativa política - eran aún capaces, merced a su alianza con los sacerdotes locales, de bloquear la mayoría de las decisiones políticas que se oponían a sus intereses. A últimos de la década de los sesenta los Taula comenzaron una feroz campaña contra los núcleos más poderosos de este segmento DC/PSI, logrando apartar a muchos de sus partidarios y obteniendo la mayoría en 1970. Virtualmente sin oposición alguna, los Taula han mantenido desde entonces un poder pleno y efectivo. ¿Por qué tuvieron éxito los Taula alli donde durante 25 años otros grupos políticos habían fracasado?

En los primeros años de su cartera comercial, hasta aptoximadamente 1965 , los Taula dieron trabajos a corto y largo plazo a un grupo de parientes a los que llamaré parientes de nivel inferior. ${ }^{27}$ Estos individuos eran parientes cercanos, principalmente hermanos o primos carnales de dos de las esposas de los hermanos Taula, que pueden ser descritos como parientes de nivel inferior porque estaban relativamente indefensos con respecto a sus patrones: desde que los tres hermanos y sus familias constituyeron un grupo afín fuerte e internamente cohesivo, este nivel inferior ya no dispuso de autoridad para apelar en casos de trato injusto, conflictos laborales, etc. Debemos mencionar que hasta esa época los Taula habían virtualmente ignorado por completo a sus propios parientes consanguíneos; muy pocos trabajos o favores comerciales habían sido concedidos a sus propios parientes, tales como sus primos carnales.

Después de 1965, coincidiendo con la expansión de sus negocios y la creciente conciencia de su aislamiento político dentro de la comunidad, se produjo un cambio significativo en sus prácticas de empleo. Gran númeto de trabajos temporales y pequeños favores fueron concedidos a parientes lejanos: primos segundos o terceros matrilineales y patrilineales, y parien. tes del grupo de nivel inferior, quienes, por ese tiempo, constituían el núcleo del bando Taula.

Con la progresiva expansión de sus negocios a comienzos de la década del setenta, los Taula comenzaron a tener en cuenta también a sus propios

sorprendernos, ya que incluso en dicho período un $70 \%$ estimado de la población exa de facto analfabeta. Este aspecto se analiza fuego con más detalle.

27. Julian Pitt-Rivers considera al patronazgo como una «amistad desequilibrada», un concepto que expresa una relación similar a la de «parentesco de nivel inferior». Pitt-Rivers, The People of the Sierra (Chicago: University of Chicago Press, 1954/1961). 
parientes consanguineos. A muchos de los individuos más dinámicos se les alentó para que establecieran tiendas de comestibles, bares y mueblerías fwera de Telemula. Según mi opinión, estos parientes cercanos eran considerados como una amenaza potencial interna desde el momento en que, a diferencia del nivel inferior de parentesco, podían aplicar sanciones y apelar a la «más alta autoridad», tales como miembros de la generación parental, quienes a su vez podían bloquear los asunros relativos a la herencia o quitar el apoyo familiar a los tres hermanos. Por otra parte, en la medida en que los tres hermanos tenían un acceso similar a dicha autoridad, este parentesco cercano podía set confiado con las inversiones que los Taula realizaban en beneficio de sus parientes, como satélites fuera de Telemula.

Por otra parte, las proposiciones a parientes lejanos -a individuos que a menudo tenían una estrecha relación de parentesco con la oposición a los Taula- eran formuladas a ellos como parientes lejanos. En consecuencia, se activaron los lazos de parentesco lejano, otorgándosele una significación concreta por medio de favores, especialmente empleos. Por lo tanto, altededor del núcleo del grupo Taula, una esfera más amplía de parientes podían verse a sí mismos como «consanguíneos» y cambiar su lealtad política (y la de algunos de sus propios parientes cercanos) en favor de los Taula, quienes ahora podían movilizar un apoyo mayoritario en el pueblo y, en consecuencia, transformarse en la nueva élite local.

Para completar el panotama, debe mencionarse aquello que podríamos denominar la subélite local, un grupo de tres «tenientes» a quienes los Taula han otorgado la mayor parte de contratos municipales tecientes, especialmente en obras públicas tales como construcción de carreteras y transporte de materiales de construcción. Estos tres varones, directamente o a través de sus esposas, son parientes lejanos de los Taula y se hallan vinculados también directamente entre sí. Comienzan a asumir como segundo en el mando un importante papel oligárquico en el pueblo.

He mencionado anteriormente que las fuerzas de oposición a los Taula mantenían lazos antiguos con la Democrazia Cristiana (DC). En nuestros días esta situación no ha cambiado y estos lazos son importantes, puesto que la DC es aún hoy la fuerza dominante en Cerdeña. ¿Cómo pueden entonces los Taula funcionar como élite local si la oposición está relacionada con la fuerza política dominante de la región?

En el pasado, los Taula han sido partidarios del ala conservadora del Partito Sardo d'Azione y del pequeño Partido Republicano. Sin embargo, en 1970, cuando Bruno Taula fue elegido alcalde, los políticos y buróctatas provinciales y regionales de la DC comprendieron que los Taula representaban una nueva e importante fuerza política en la comunidad y que opera- 
ban dentro del marco de la estructura política vigente, y que ellos dependían del patronazgo en las capitales provinciales y regionales.

Por lo tanto, mientras los Taula permanecían oficialmente fueta del contexto de la DC y como antagonistas de la Iglesia, habían desarroliado rápidamente, a lo largo de los últimos años, vínculos altamente personales con importantes tecnócratas políticos que eran miembros de la DC y por lo tanto habían tenido éxito en evitar el patronazgo "oficial» de la DC extendido a las fuerzas anti-Taula por notables más antiguos. Como consecuencia, el viejo patronazgo de la DC en Telemula se ha estancado, restringido a ocasionales trabajos en el servicio de reparación de carreteras, al empleo por un hombre de negocios y político de la DC fuera de Telemula, etc.

Cabe señatar, por lo tanto, que el viejo sistema de patronazgo de la $D C$ en Telemuia está cualitativamente al nivel de los otros partidos, aunque es comparativamente más hábil: el electorado socialista, neofascista, e incluso comunista, están relacionados con las personalidades notables de la región, especialmente abogados y contratistas en las ciudades principales, quienes prestan servicios ocasionales a cambio de apoyo electoral. En consecuencia, lo que es importante es que los Taula controlan los lazos significativos, los lazos con los butócratas financieramente solventes que disponen de los principales fondos del gobierno, fondos para el mejoramiento de la infraestructura turística, de la economía local y de otros sectores de la infraestructura. Este tipo de fondos desembocan en empresas controladas por los Taula. Ciertamente no es exagerado decir que, a lo largo de los últimos veinte años, los Taula han recibido muchos más fondos estatales, beneficios de impuestos, etc., por sus empresas comerciales que todos los otros telemuleses juntos.

Si comparamos a los Taula, como nueva élite local, con la élite que se había establecido en los ptimeros años de existencia del Estado italiano, surge una serie de puntos interesantes.

Familismo amoral y exclusivista. Ahora menos que nunca puede ser descrita la élite local como exclusivista en el sentido dado por Pinna al término. Los Taulas consideran cuidadosamente quiénes son los que deberían recibir los empleos y ottos favores, pero los empleos se distribuyen ampliamente a través de la comunidad. ${ }^{28}$ Los Taula utilizan la cualidad

28. Hasta el punto en que algunos de los parientes cercanos de Bruno Taula se quejan de ser techazados en favor de otros. Sin embargo, un análisis cuidadoso revela que muy pocos trabajos estaban destinados a aquellos individuos que no eran parientes de los Taula. 
conectiva inherente a los lazos de parentesco a fin de vincular amplios segmentos de la población del pueblo con ellos. De este modo, ellos reciben el apoyo político necesatio para expandir su papel económico. Por lo tanto, la concepción de Banfeld sobre el familismo se aplica efectivamente a los Taula, como nueva élite local, pero no a la comunidad toda.

El parentesco como superestructura dominante. Con la decadencia de la vieja élite local, el parentesco asumió un papel principal en la estructu. ración de la economía. Actualmente, al menos en la esfera de los Tauia, el papel del parentesco se ha vuelto dominante. Tanto en su familia inmediata como asimismo en una esfera más amplia, las decisiones económicas son dirigidas por consideraciones de parentesco.

La élite local y el Estado. Mientras que tanto la vieja élite local como los Taula están firmemente enraizados en la estructura local de parentesco, los Taula no personifican al Estado de la forma en que lo hicieron los Sanna. En tanto que la supervivencia de los Taula como élite local depende de su preminencia política, son antes que nada representantes de la economía capitalista y de la clase capitalista. La vieja élite local estaba integrada por políticos notables, religiosos $e$ intelectuales, sobre cuya base ellos construyeton su imperio económico; Ios Taula son fundamentalmente capitalistas y su posición política es dependiente. Además, en el área rural la vieja élite local sólo esporádicamente estableció telaciones duraderas, tales como matrimonios cruzados con la clase dirigente sarda, en Cagliari o Sassari. Sin embargo, contrastando con esta vieja élite local, los Taula y grupos similates en otros pueblos mantienen entre ellos lazos viables y especialmente con la clase alta en las ciudades. Los Taula, por ejemplo, hacen hincapié en el hecho de enviar a sus hijos a internados de élite en Cagliari en lugar de hacerlo a un liceo de una ciudad vecina, como hicieron los Sanna en el pasado y aún lo hacen hoy.

Por otra parte, la vieja elite local mantenía un exclusivo monopolio de acceso al Estado. Con el establecimiento de una estructura de partido después de 1945, otros grupos también obtuvieron cietto acceso a aquellas instituciones políticas externas. Estas instituciones, no obstante, ofrecen simplemente diversas formas de bienestar, mientras que los Taula mantienen un monopolio de acceso a aquellas instituciones políticas que tienen un papel fundamental dentro de la economía capitalista. ${ }^{29}$

29. Dos ejemplos itustran esta nueva cualidad de monopolización del acceso. Los miembros de las fuerzas anti-Taula habían tenido algún éxito en los tribunales, los que 
El fenómeno de las élites locales nueva y vieja —en Telemula, el gobierno autocrático de los Sanna durante tres cuartos de siglo y recientemente el poderoso dominio de los Taula sobre los asuntos del pueblonos plantea este interrogante: ¿por qué se hizo posible este interregno ligeramente caótico entre 1945 y 1970 , y por qué, durante esa época, no surgió una élite gobetnante de los grupos que detentaban el poder nominal en Telemula? Un análisis de la cantidad de miembros del consiglio comunale (consejo comunal) y un repaso a los antecedentes de los distintos alcaldes durante dicho periodo, quienes eran mucho más representativos del telemulense común que de los Sanna o de los Taula, nos plantea claramente esta cuestión: ya fuesen pastores o agricultores, estos individuos pertenecian al sector económico tradicional de la comunidad.

$\mathrm{Ni}$ su concepción «moderna» los habilitaba para formar una élite local nueva y estable - algunos de los varones habian intentado desarrollar actividades similares a las de los Taula en el campo comercial, pero fracasaron- ni tampoco su política progresista o conservadora, como miembros de la DC o de partidos del ala izquierda, hizo que dicho papel se concretara. El solo hecho de que se hallan atados a sectores económicos tradicionales y estancados, sectores que han recibido poca o ninguna ayuda del gobierno, mientras que billones de liras etan derramados sobre otros, hizo imposible que cualquier grupo en el sector pastoril o agrícola obtuviera un firme control político.

Solamente a fines de la década de los sesenta, durante un corto período en el que fue elegido alcalde un perito agrario (especialista agrícola) de origen telemulense, pero que vivía fuera del pueblo, pareció como si desde este sector tradicional pudiese formarse una nueva élite local alrededor de dos cooperativas agrícolas que habian sido propuestas por el alcalde. Pero para esa época los Taula ya eran demasiado poderosos. Logtaron volcar la opinión pública contra tales «favoritismos» y finalmente impidieron el

habían revocado algunas decisiones municipales que se referfan a cliestiones menores acerca de la propiectad y otros tuvieron a los tribunales de su parte en un caso en el que un aliado intimo de los Taula insultó a un oponente de la familia. Por otro lado, la persona encargada de coordinar la planificación de una nueva cooperativa y que se hallaba estrechamente vinculada a un alcalde anterior a 1970 (opuesto a los Tavia) descubrió que después de la elección de Bruno Taula como alcalde, los abogados y administradores de Cagliari (capital de la región de Cerdeña) zechazaton cualquier trato con él. 
éxito de estos planes. Los presuntos miembros de estas dos cooperativas estaban todos relacionados por lazos de parentesco y los Taula comprendieron claramente que semejante cooperación económica dentro de un contexto de parentesco hubiese dado lugar a una seria y sostenida oposición a sus propios objetivos políticos. ${ }^{30}$

30. Habría que mencionar aquí brevemente el análisis que hace Alex Weingrod acerca del patronazgo en Surughu, una comunidad próxima a Oristano, en la región centro-occidental de la isla. Véase «Patron, Patronage and Political Parties». Comparative Studies in Society and History, vol. 10 (1967-1968): 376-400. A pesar de que esta comunidad no forma parte de la Barbagia, y aunque parece que la agricultura juega un papel más importante que en aquellas comunidades a las que he estudiado, existen algunos paralelos notables entre los hallazgos de Weingrod y este análisis. Weingrod está interesado en el patronazgo y ve dos estadios históricos durante los cuales asume diferentes formas. En su primer estadio, entre 1870 y 1922, el patronazgo toma la forma de una «jerarquía informal - una especie de trama amistosa basada en la influencia» (ibid., p. 379) y él llama al patronazgo del segundo estadio, o sea el período desde 1922 - incluyendo n.b. tanto al fascismo como a la nueva república posterior a 1945- «sistema de patronazgo de partido político» (ibid., p. 381). En el primer estadio, Surughu estaba caracterizada por los patrones: varios terratenientes, «dos familias de abogados, el sacerdote del pueblo $\mathrm{y}$, fundamentalmente, el sindaco del pueblo» (391). En términos más específicos, hay líderes políticos del pueblo que tienen «estrechos lazos de beneficio mutuo» (392) con terratenientes, y los terratenientes a su vez tienen una clientela entre los mezzadri que trabajan en sus tierras. Por lo tanto, hay dos familias de abogados que mantienen lazos políticos fuera del pueblo y «sus servicios eran requeridos con frecuencia (392); por otra parte, el sacerdote del pueblo que asumió un papel activo en los asuntos locales y emplé sus «canales de información” para asistir a sus feligreses. Weingrod describe a esta gente como «influyentes» que a menudo «desempeñan el papel de patrón», pero la fuerza política principal del pueblo, en el estadio uno, era un alcalde en particular que, aliado con varios terratenientes, pudo surgir como el «amo local».

La caracterización que hace Weingrod de este primer estadio señala -coincidiendo con mis hallazgos - el importante papel desempeñado por los terratenientes y la Iglesia en el período anterior a la Primera Guerra Mundial. Lo que sin embargo se halla totalmente negado en este punto es la forma en que estos distintos patrones se hallan relacionados entre sí -si son antagonistas o están ligados por el parentesco, si tienen intereses económicos en común, etc., y, aparte de los terratenientes, no obtenemos una idea distinta sobre la base económica de numerosas tamilias - ¿cómo, por ejemplo, pudieron las dos familias de abogados afrontar una educación universitaria para sus hijos? En otras palabras, Weingrod conceptualiza a los patrones, pero es incapaz de analizarlos como parte de la élite local, con ciertos intereses de clases y tareas políticas específicas y lazos que se originan fuera de la comunidad.

En la primera parte de su segundo estadio, el fascismo, observa un cambio significativo. Con mucha percepción señala la creciente incorporación de la comunidad local a las estructuras del Estado, una vinculación más estrecha entre la comunidad local y «los sistemas políticos, económicos y sociales de toda la islaw (393). En este proceso tanto los terratenientes y el alcalde, como «amo local», parecen desvanecerse; el secre- 
Familismo y patronazgo, sistemas de poder en Cerdeña

\section{Conclusiones}

Resumiendo, que tanto en la Barbagia como probablemente en otras zonas mediterráneas con relaciones de producción y estructuras de Estado

tario local del Partido Fascista y las dos familias de abogados, ahora fntimamente vinculados al Partido Fascista, asumen un papel de patronazgo, pero las decisiones importantes se toman en círculos de partido exteriores a la comunidad, un hecho que parece deprimir el papel eminente desarrollado por los patrones locales.

En la concepción de Weingrod muchos de los procesos de desarrollo iniciados por el fascismo fueron «magnificados» en la república posterior a la guerra: «Las brechas sociales y también culturales entre el sistema del pueblo y la región y la nación se han cerrado paulatinamente, y de muchas e importantes maneras los habitantes del pueblo participan ahora de la "sociedad de masas" y la "cultura de masas" de la Italia contemporánea» (396). Por otra parte, el gobierno unipartito del Fascismo, sostiene Weingrod, ha sido reemplazado por el papel patronal de un sistema multipartito. Si comparamos esta situación con la evidencia extraída de Telemula, encontramos algunas divergencias significativas. En primer lugar, a pesar de una interrupción temporal a raíz de la rebelión anti-Sanna (ver nota 22) y a pesar de las simpatías fascistas de un alcalde opuesto a la vieja élite local, los Sanna pronto recuperaron su posición dominante en el pueblo a fines de la década de 1920, como podestd fascista o como segretario prefettizio, vinculados ahora, por supuesto, al Partido Fascista. Mientras que es totalmente correcto afirmar que el sistema político ha sido manifiestamente alterado después de 1922, con una penetración mucho más efectiva en el nivel local por el aparato del Estado, Weingrod no ve en absoluto las continuidades, la permanente dominación ejercida por la vieja clase alta local, un punto que seguramente podría haber sido señalado también para el caso de Surughu. (El mismo Weingrod señala el papel permanente desempeñado por las familias de abogados.)

Por otra parte, incluso desde una perspectiva «pluralista», es naturalmente un grueso error el igualar, después de la guerra, los recursos aprovechables por la democrazia cristiana con los de los comunistas, socialistas y otros partidos políticos. No existe una seria competencia entre «don Camilo» y «Poppone», especialmente en el sur y en los primeros años de la década de 1960, época en la que la DC controla la Cassa per il Mezzogiorno que, en palabras de La Palombara, es una sorganización gigantesca de patronazgo».

Puede muy bien decirse que, desde el establecimiento de la República, «ninguna figura ha dominado la política de Surughu (397; el subrayado es mío). Hasta 1970, esta afirmación también puede aplicarse al caso de Telemula. Pero, ¿existía realmente una alternación en el poder, o se lo compartía libremente, en el pueblo estudiado por Weingrod? Lo dudo mucho. Una conclusión implícita como ésta es completamente ciega a la estructura de clase que impera en el ambiente rural de Cerdeña.

Se puede presumir, entonces, que Weingrod encuentra que los nuevos amos de partido que manejan el patronazgo partidario «probablemente atiendan mejor a sus "electores" de lo que puede hacerlo el patrón con sus "clientes"» (384); por lo tanto, el pluralismo político puede «superar» las tradicionales «diferencias de status» y ayudar a hacer más relevantes los «nuevos valores igualitarios» (384) y arrojar a un proceso emigratorio a miles de varones y mujeres del sur. 
similares, encontramos una élite local incrustada en una estructura de parentesco hipostatizada —una estructura que ejetce un control significativo sobre las relaciones de producción - que monopoliza el acceso a las instituciones clave del Estado.

Naturalmente debemos ser prudentes cuando inferimos a partir de las diferencias entre las élites locaies vieja y nueva en Telemula a Cerdeña en general. Por ejemplo, en otras comunidades podemos hallar no ya una sucesión de diferentes grupos de parentesco en cuanto a las élites locales, sino una transformación de un grupo de parentesco perteneciente a la «vieja» élite en uno de la «nueva». A pesar de ello, yo diría que típicamente el monopolio de acceso ejercido por la nueva élite se halla mitigado por la estructura partidaria que permite el patronazgo marginal fuera del contexto de la élite local. Por otra parte, en contraste con la vieja élite que aparecía como una representación personificada del Estado, la nueva élite aparece como el representante y miembro joven de la clase capitalista. Mientras que la vieja élite local presentaba una tendencia a encapsularse y confiar en sus lazos con ciertas personalidades notables en la capital, la nueva élite se halla vinculada más efectivamente a sus equivalentes en ciudades vecinas, a los modernos tecnócratas de oficina y a la clase alta de la isla.

Por último, debemos agregar la cuestión de cómo puede evolucionar en el futuro la estructura social de estas comunidades. He intentado mostrar que no toda la estructura social es familística, solamente lo es la elite local, o sea, los lazos de parentesco que alguna vez constituyeron las relaciones de producción han asumido un poderio propio y están concentrados en una minoría pequeña y poderosa. Actualmente, con la destrucción de la economía pastoril y campesina tradicional y la parálisis de la economía tural, el parentesco como viejo principio organizador ha perdido su significado. Trabajadores emigrantes se emparentan con familias que constituyen un anatema para sus hermanos y parientes. Los estudiantes, maestros y profesionales locales, marginales al pueblo, ya no saben exactamente quiénes son sus primos segundos o terceros. La masa restante de aquellos que realizan trabajos precarios en el pueblo, independientemente de sus lazos familiares, se asocian con o contra la élite local, con o contra sus grupos de parientes, depende de cuál de ellos tiene más posibilidades de ayudarles a obtener un trabajo o un ingreso extra.

Éste es el contraste, por lo tanto, entre la concentración de lazos de parentesco en la cima y la negación de la solidaridad parental en el fondo de la estructura social. Yo creo que es esta erosión de las solidaridades parentales particularizadas en el fondo la que podría producir solidaridades ampliamente fundamentadas de campesinos y pastores y, en consecuencia, 
Familismo y patronazgo, sistemas de poder en Cetdeña acabar con la élite local familística que ha abortado todos los intentos por transformar la economía rural.

Y. Michal BodemanN

Department of Sociology

University of Toronto

563 Spadina Ave.

Toronto, Ontario M5S $1 \mathrm{A1}$

Canadá 